\title{
EVALUATION DE LA PERTE D'OEUFS LORS DE L'INCUBATION CHEZ NEPHROPS NORVEGICUS (L.) DANS LA RÉGION SUD-BRETAGNE, FRANCE
}

\author{
PAR \\ YVON MORIZUR \\ Centre Océanologique de Bretagne, B.P. 337, 29273 Brest, France')
}

\section{INTRODUCTION}

L'incubation des oeufs chez la langoustine, Nephrops norvegicus (L., 1758), dure 7,5 mois environ dans le Golfe de Gascogne (Fontaine \& Warluzel, 1969). Au cours de cette période, les oeufs passent par différents stades qui ont été décrits par Figueiredo Barraca (1963). Selon la quantité de vitellus présent dans l'oeuf et l'aspect externe de l'embryon, ces auteurs ont déterminé 5 stades, de durée inégale, pour une durée totale de 6,5 mois dans les eaux portugaises: stade A (durée 2,5 mois), stade B (durée 1 mois), stade C (durée 1 mois), stade $D$ (durée 1 mois), stade $E$ (durée 1 mois).

Les langoustines vivent sur des fonds vaseux et habitent des terriers où elles passent une partie de leur temps (Farmer, 1975). Il est généralement observé que la langoustine "grainée" disparait des captures presque aussitôt après la ponte et passe alors la plus grande partie de son temps dans le terrier (Thomas \& Figueiredo, 1964; Farmer, 1975). Les femelles ovigères se nourrissent moins fréquemment que les femelles non ovigères (Dunthorn, 1967). Cette diminution de la prise alimentaire a également été décrite chez Homarus gammarus (L.) par Brandford (1979).

Comme les langoustines ovigères sont très peu nombreuses dans les captures au cours des derniers stades d'incubation, les études de fécondité ont été menées essentiellement à partir des ovaires. C'est ainsi que Fontaine \& Warluzel (1969) ont évalué la fécondité de Nephrops norvegicus dans le Golfe de Gascogne. Aucune évaluation de la fécondité n'a été faite en fin d'incubation. Figueiredo \& Nunes (1965), au Portugal, ont estimé que la perte d'oeufs au cours de la ponte et de l'incubation était de $75 \%$ quelle que soit la taille de la femelle. La présente étude cherche à évaluer, chez la langoustine Nephrops norvegicus, la perte d'oeufs qui se produit, dans le Golfe de Gascogne, lors de l'incubation. 


\section{MATERIEL ET METHODES}

Les oeufs, une fois détachés de l'abdomen, sont placés à l'étuve à $100^{\circ} \mathrm{C}$ durant dix minutes. Ce procédé a pour but de faciliter leur séparation pour le comptage. Les comptages sont effectués à la loupe binoculaire sur une cuve Dollfus. Lorsque le nombre d'oeufs n'excéde pas la valeur de 1000 , la totalité d'entre eux est comptée. Dans les autres cas, nous fractionnons les oeufs par pesée de façon à conserver une précision de l'ordre de $5 \%$ dans les estimations.

Nous utilisons les stades d'incubation de Figueiredo \& Barraca (1963) auxquels nous ajoutons le stade $\mathrm{O}$ correspondant aux oeufs en fin de maturation ovarienne. Toutefois, nous choisissons comme stade de fin d'incubation le stade $\mathrm{D}$ au lieu du stade $\mathrm{E}$ pour éviter d'effectuer des comptages pour les femelles dont les oeufs sont partiellement éclos. La détermination du stade est effectuée à la loupe binoculaire pour chacune des langoustines.

La mesure de la longueur du céphalothorax des femelles "grainées" est prise au pied à coulisse au $1 / 10$ è de mm près. Nous limitons notre étude aux tailles comprises entre 22,5 et $27,5 \mathrm{~mm}$ en raison des faibles effectifs situés lors de ces limites de classe. C'est dans cet intervalle de taille que la pente de la relation "nombre d'oeufs en fonction de la taille de la femelle" est la plus faible (Fontaine \& Warluzel, 1969; Figueiredo \& Nunes, 1965). Mais, pour limiter les effets du facteur taille sur les estimations de fécondité, nous créons deux sous-groupes $22,5-25 \mathrm{~mm}$ et $25-27,5 \mathrm{~mm}$.

Les pertes sont évaluées suivant deux méthodes:

1. Nous calculons la perte d'oeufs entre le stade d'incubation A et le stade d'incubation $D$. Cette perte est ensuite extrapolée linéairement à toute la durée d'incubation. Pour chacune des classes, les deux échantillons A et D, correspondant respectivement aux stades d'incubation $A$ et $D$, sont indépendants et ne possèdent pas le même effectif. Pour comparer, dans chacune des classes, les moyennes de chacun des groupes $A$ et $D$, nous utilisons l'analyse de variance à deux facteurs avec répétitions, après avoir testé l'homogénéité des variances à l'aide du test de Bartlett (Snedecor \& Cochran, 1967).

2. Nous calculons la perte d'oeufs entre le stade $O$ (fin de maturation ovarienne) et le stade $\mathrm{D}$. Cette perte est ensuite extrapolée linéairement à toute la durée d'incubation. Nous estimons, d'après nos observations de ponte au laboratoire (données non publiées), que la perte d'oeufs lors de la ponte est très faible (inférieure à $3 \%$ ). La quantité d'oeufs demeurant dans les ovaires après la ponte peut être considérée insignifiante selon nos observations sur des langoustines prélevées dans le Golfe de Gascogne. Pour évaluer le nombre d'oeufs au stade $O$, nous nous référons aux travaux que Fontaine \& Warluzel (1969) ont réalisés sur la langoustine du Golfe de Gascogne. Selons ces auteurs le nombre d'oeufs $(F)$ contenus dans les ovaires serait une fonction allométrique de la longueur du céphalothorax (L) de la femelle: $F=0,108 \mathrm{~L}^{2.797}$.

Les deux méthodes reposent sur l'hypothèse, soutenue par Figueiredo * Nunes (1965), selon laquelle la perte d'oeufs est une fonction linéaire du temos. 
Les langoustines "grainées" en stade d'incubation A ou D ont été capturées au chalut lors de pêches dans la région de Sud-Bretagne respectivement en septembre 1978 et en février-mars 1979. Elles ont été prélevées par les pêcheurs et fixées dans l'alcool à $70^{\circ}$. Le stade d'incubation a été identifié au laboratoire.

\section{RESULTATS}

1. Les variances du nombre d'oeufs pour chacune des 4 cellules ne sont pas significativement différentes au seuil de $5 \%$. L'intéraction des facteurs n'est pạs significative, tandis que la différence entre les moyennes l'est, pour chacun des facteurs (test $\mathrm{F}$ au seuil de $5 \%$ ). Nous pouvons donc distinguer deux classes de taille dans lesquelles les moyennes sont significativement différentes et considérer que la perte d'oeufs en valeur absolue est indépendante de la taille. Les résultats (moyennes, variances, valeurs de F) ainsi que les taux de perte sont portés dans le tableau I.

\section{Tableaux I}

Résultats obtenus par la première méthode

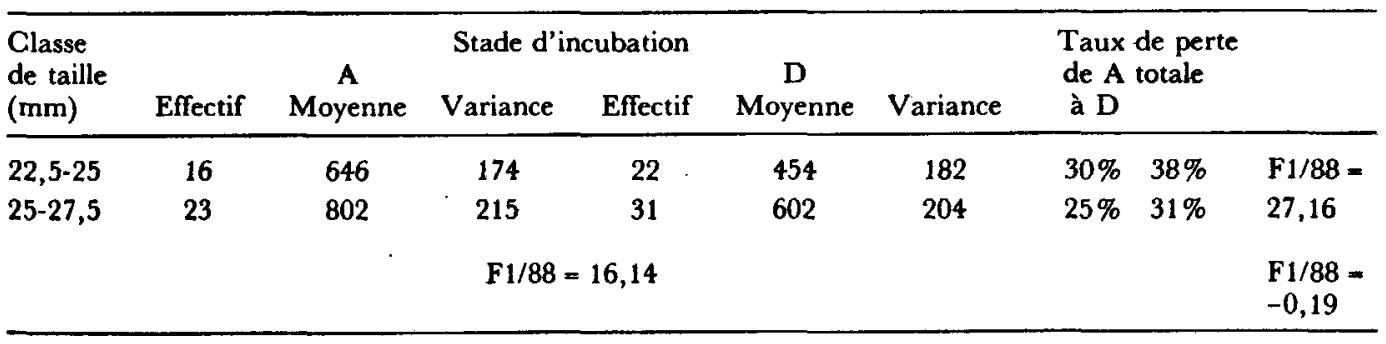

F1/88 (seuil de $5 \%$ ) $=3,92$.

Le taux de perte des oeufs entre les stades $A$ et $D$ correspond à celui d'une incubation de 6 mois. Nous avons ensuite estimé le taux de perte pour une incubation d'une durée totale de 7,5 mois.

Cette première méthode indiquerait donc une perte d'oeufs durant l'incubation de 30 à $40 \%$ pour les tailles étudiées.

2. Pour calculer le nombre d'oeufs contenus dans les ovaires, nous n'avons pas utilisé le centre de chacune des 2 classes de tailles. Nous avons préféré nous servir de la moyenne arithmétique des longueurs de céphalothorax des langoustines possédant des oeufs de stade $D$. Ces moyennes sont 24,1 et $26,4 \mathrm{~mm}$. La durée d'incubation du stade $O$ au stade $D$ est estimée à 6,5 mois. A partir de la perte d'oeufs correspondant à cette période, nous avons calculé la perte pour une durée d'incubation totale de 7,5 mois. Ces résultats sont portés dans le tableau II.

Cette dernière méthode indiquerait, pour les tailles considérées, une perte d'oeufs durant l'incubation de l'ordre de 45 à $50 \%$. 
Résultats obtenus par la deuxième méthode

\begin{tabular}{lccccc}
\hline $\begin{array}{l}\text { Classe de taille } \\
(\mathrm{mm})\end{array}$ & \multicolumn{2}{c}{ Nombre d'oeufs moyen } & \multicolumn{2}{c}{ Taux de perte } \\
& Stade O & Stade D & de O à D & Totale \\
\hline $22,5-25$ & 790 & 454 & $43 \%$ & $49 \%$ \\
$25-27,5$ & 1020 & 602 & $41 \%$ & $47 \%$ \\
\hline
\end{tabular}

\section{DISCUSSION}

Les pertes évaluées selon la première méthode sont plus faibles que celles obtenues par la seconde. La différence ne proviendrait pas uniquement de la perte qui se produit lors de la ponte et que seule la deuxième méthode prend en compte. La première méthode nous semble minimiser les pertes. Cette minimisation serait due au fait que le taux de perte d'oeufs lors de la pêche des langoustines au chalut n'est pas le même pour les deux stades d'incubation considérés $\mathrm{A}$ et $\mathrm{D}$. En effet, le taux de perte dû à l'engin de pêche est vraisemblablement plus élevé pour le stade $A$ que pour le stade $D$. Les résultats les plus réalistes seraient donc obtenus par la deuxième méthode. Ils indiquent une perte d'oeufs durant l'incubation de l'ordre de 40 à $50 \%$ pour les tailles étudiées.

Nos résultats sont très différents de ceux obtenus par Figueiredo Nunes (1965). Selon ces auteurs, la perte lors de l'incubation serait de $75 \%$ et la perte d'oeufs due à l'action de pêche ne serait pas une source de biais. Il convient de remarquer que Figueiredo \& Nunes (1965) ont sélectionné des langoustines qui n'avaient pas été lésées par le chalut, précaution que nous n'avons pas prise. Toutefois, la différence entre nos résultats et les leurs nous parait difficilement explicable par le seul dispositif expérimental. Elle ne s'explique pas non plus par les durées d'incubation quelque peu différentes. Celles-ci auraient dû créer une différence dans le sens contraire de celui observé. Il s'agirait donc de différences biologiques.

La perte d'oeufs lors de l'incubation serait un facteur de régulation de la densité du stock et un des mécanismes de fonctionnement de la relation stockrecrutement. Selon Chapman \& Howard (1979), la sortie hors des terriers est une activité liée essentiellement à l'alimentation. Nous pensons que c'est lors de cette activité que la prédation sur les oeufs s'exerce de façon importante par les crabes, crevettes... Ces dernières se sont révélées être, en laboratoire, de grands prédateurs d'oeufs (observation personnelle). De plus, le cannibalisme au niveau des oeufs existe peut-être chez la langoustine. Lorsque les langoustines sont nombreuses sur les fonds, elles doivent passer plus de temps hors du terrier pour la recherche de nourriture. La prédation et le cannibalisme sur les oeufs sont alors favorisés lorsqu'elles sont "grainées". A ce niveau, il convient de noter que l'activité de pêche agit de deux manières convergentes. La diminution de densité qu'elle provoque tend à diminuer la perte d'oeufs lors de 
l'incubation. Les rejets à la mer, qui sont une source de nourriture non négligeable (Warren, 1976), favorisent également une diminution de la prédation sur les oeufs.

La différence observée entre les taux de perte d'oeufs lors de l'incubation proviendrait de la mortalité totale qui ne serait pas égale pour les deux stocks. L'effort de pêche déployé est, vraisemblablement, plus important en SudBretagne que dans les eaux portugaises. Il n'est pas impossible aussi que les fonds soient differents quant à leur richesse en prédateurs.

Le taux de perte est-il constant quelle que soit la taille de la femelle comme le prétendent Figueiredo * Nunes (1965) ou diminue-t-il en fonction de la taille comme le suggèrent nos résultats (tableaux I et II)? La dernière hypothèse ne nous parait pas invraisemblable car les grosses langoustines doivent être plus aptes à défendre leurs oeufs contre les prédateurs.

Chez Nephrops norvegicus, la perte d'oeufs lors de l'incubation serait plus faible dans le Golfe de Gascogne que dans les eaux portugaises. Elle serait due à un effet régulateur lié à l'écosystème et à la pêche.

\section{REMERCIEMENTS}

Nous tenons ici à remercier Jean Guillaume et Gérard Conan pour leurs judicieuses critiques ainsi que $A$. Guénolé et $M$. $H$. Omnès pour leurs précieuses aides techniques.

\section{SUMMARY}

- Loss of eggs during the incubation period in ovigerous females of Nephrops noroegicus from the Bay of Biscay is calculated using two different methods. The first calculates the egg loss from incubation stage $A$ to stage $D$. The second calculates the loss between the end of ovary maturation and incubation stage $\mathrm{D}$. The first method may contain a bias which decreases the loss estimation: loss of eggs during trawling may be higher in stage $A$ than in stage $D$. In the Bay of Biscay, the loss of eggs in ovigerous females of Nephrops norvegicus would be about $45-50 \%$ for the entire incubation period. Our result is quite different from the $75 \%$ obtained by other workers in Portuguese waters.

Loss of eggs during incubation which may be due mainly to predation may be a means of density regulation (stock-recruitment relationship).

The differences observed in loss of eggs between the South-Brittany area and Portuguese waters may result from differences in fishing mortalities or in the density of predators. -

\section{BIBLIOGRAPHIE}

Brandford, J. R., 1979. Locomotor activity and food consumption by the lobster Homarus gammarus. Mar. Behav. Physiol., 6: 13-24.

Chapman, C. J. F. G. Howard, 1979. Field observations on the emergence rhythm of the Norway lobster Nephrops norvegicus, using different methods. Mar. Biol., 51: 157-165.

Dunthorn, A. A., 1967. Some observations on the behaviour and development of the Norway lobster. I.C.E.S., (K) 5: 1-11.

Farmer, A. S. D., 1975. Synopsis of biological data on the Norway lobster Nephrops norvegicus (Linnaeus, 1758). F.A.O. Fisheries Synopsis, 112: i-iv, 1-97.

Figueiredo, M. J. I. F. Barraca, 1963. Contribuiçao para o conhecimento da pesca e da biologia da lagostim (Nephrops norvegicus L.) na costa portuguesa. Notas Estud. Inst. Biol. mar., 28: 1-45. 
Figueiredo, M. J. M. C. Nunes, 1965. The fecundity of the Norway lobster, Nephrops norvegicus (L.) in portuguese waters. I.C.E.S., Shellfish Committee, 34.

Fontaine, B. N. Warluzel, 1969. Biologie de la langoustine du Golfe de Gascogne Nephrops norvegicus (L.). Rev. Trav. Inst. Pêches marit., 33(2): 223-246.

Snedecor, G. W. W. G. Cochran, 1967. Statistical methods, (ed. 6): 1-593. (Iowa State University Press, Ames, Iowa, U.S.A.).

Thomas, H. J. M. J. Figueiredo, 1965. Seasonal variations in the catch composition of the Norway lobster, Nephrops norvegicus (L.) around Scotland. Journ. Cons. perm. int. Explor. Mer, 30: 75-85.

WARREN, P. J., 1976. Some observations on fishing practice and fish predation as factors affecting a Nephrops stock. I.C.E.S., (K) 13: 1-6. 